\section{International Scientific Journal Theoretical \& Applied Science}

p-ISSN: 2308-4944 (print) e-ISSN: 2409-0085 (online)

Year: $2016 \quad$ Issue: $12 \quad$ Volume: 44

Published: 8.12.2016 http://T-Science.org
Aleksey Aleksandrovich Lukyanov

Post-graduate student,

Lead engineer of laboratory "Automobile Technologies" of Togliatti State University, Togliatti a.lukyanov@tehnomasch.ru

Olesya Olegovna Levitskih

Engineer of laboratory "Automobile Technologies" of Togliatti State University, Togliatti

SECTION 7. Mechanics and machine construction.

\title{
ALGORITHM FOR CALCULATING THE TWO-DIMENSIONAL ROUGHNESS PARAMETERS IN THREE-DIMENSIONAL MODEL OF PART'S SURFACE
}

Abstract: In this paper the algorithm for calculating two-dimensional roughness parameters in threedimensional model of the part's surface is presented. The block diagram and a basic steps of the main geometrics calculation are given.

Key words: microrelief, simulation, geometrics, profilogram.

Language: Russian

Citation: Lukyanov AA, Levitskih OO (2016) ALGORITHM FOR CALCULATING THE TWODIMENSIONAL ROUGHNESS PARAMETERS IN THREE-DIMENSIONAL MODEL OF PART'S SURFACE. ISJ Theoretical \& Applied Science, 12 (44): 1-5.

Soi: http://s-o-i.org/1.1/TAS-12-44-1 Doi: crossef http://dx.doi.org/10.15863/TAS.2016.12.44.1

\section{АЛГОРИТМ РАСЧЕТА ДВУХМЕРНЫХ ПАРАМЕТРОВ ШЕРОХОВАТОСТИ ПО ТРЕХМЕРНОЙ МОДЕЛИ ПОВЕРХНОСТИ ДЕТАЛИ}

Аннотация: Рассмотрен алгоритм, предназначенный для расчета двухмерных параметров шероховатости по трехмерной модели поверхности детали. Приведена блок-схема алгоритма, показаны основные этапы оценки основных параметров геометрии.

Ключевые слова: микрорельеф, моделирование, геометрические параметры, профилограмма.

\begin{tabular}{lrrr}
\multicolumn{3}{c}{ Introduction } & \\
Современное & \multicolumn{2}{c}{ машиностроение } \\
характеризуется & постоянным & повышением \\
требований & к & геометрическим & параметрам \\
качества & поверхностей & деталей & после \\
механической & обработки, & а & также \\
микрогеометрии & наиболее & ответственных
\end{tabular}
участков $[1,2,3]$.

\section{Materials and Methods}

Компьютерное моделирование процесса обработки позволяет снизить издержки при проектировании новых изделий и разработке технологических процессов [4]. При этом требуется анализ и оценка полученных в процессе моделирования результатов $[5,6]$.
Разработанный алгоритм предназначен для расчета параметров шероховатости в выбранном сечении и их вывод в стандартных величинах.

Далее будут проиллюстрированы типовые шаги алгоритма.

После компьютерного моделирования механической обработки поверхность приобретает сложный стохастический профиль. Данные, получаемые с помощью алгоритма о поверхности, представляют собой матрицу в которой в каждой ячейке записана высота отдельной микронеровности. Если попытаться построить трехмерные кривые в двухмерной проекции то получится результат, представленный на рисунке ниже (рис. 1). 


\begin{tabular}{l|lrl|l|ll} 
& ISRA (India) & $=\mathbf{1 . 3 4 4}$ & SIS (USA) & $=\mathbf{0 . 9 1 2}$ & ICV (Poland) & $=\mathbf{6 . 6 3 0}$ \\
Impact Factor: & ISI (Dubai, UAE) $=\mathbf{0 . 8 2 9}$ & PUHU (Russia) $=\mathbf{0 . 2 3 4}$ & PIF (India) & $=\mathbf{1 . 9 4 0}$ \\
& GIF (Australia) & $\mathbf{0 . 5 6 4}$ & ESJI (KZ) & $=\mathbf{1 . 0 4 2}$ & IBI (India) & $\mathbf{4 . 2 6 0}$
\end{tabular}

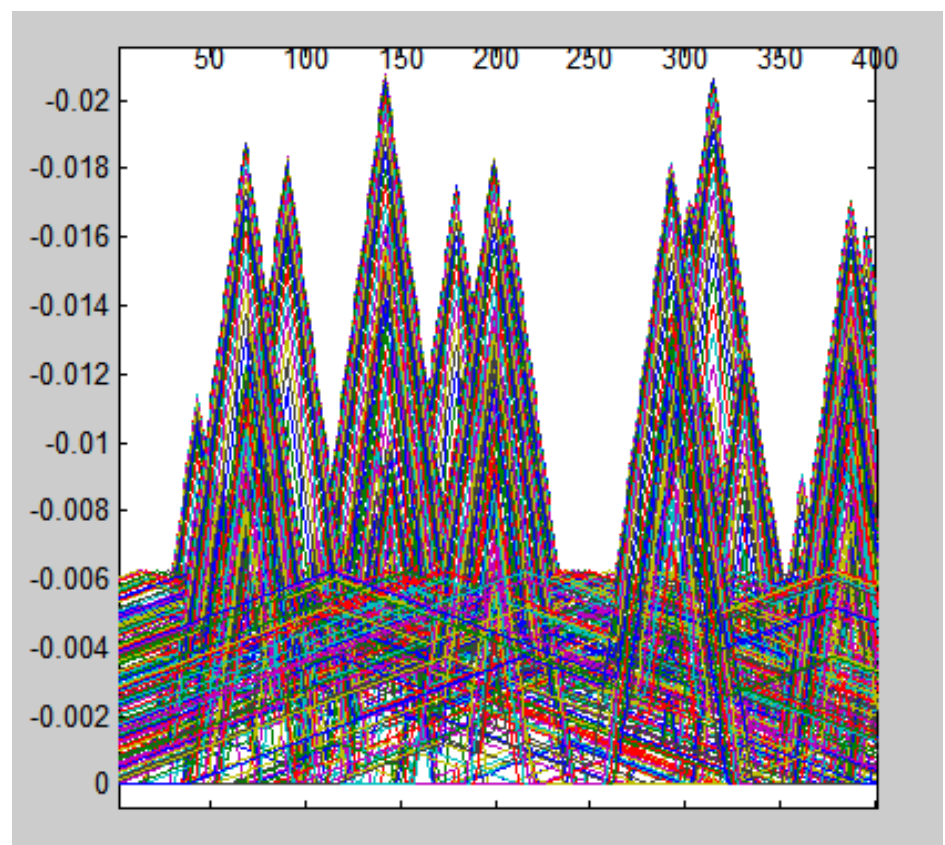

Рисунок 1 - Неотфильтрованные профили поверхности

Для получения численных двухмерных показателей необходимо выделить их в отдельную огибающую (профилограмму), по аналогии с реальным процессом фильтрации данных в устройствах типа профилограф.

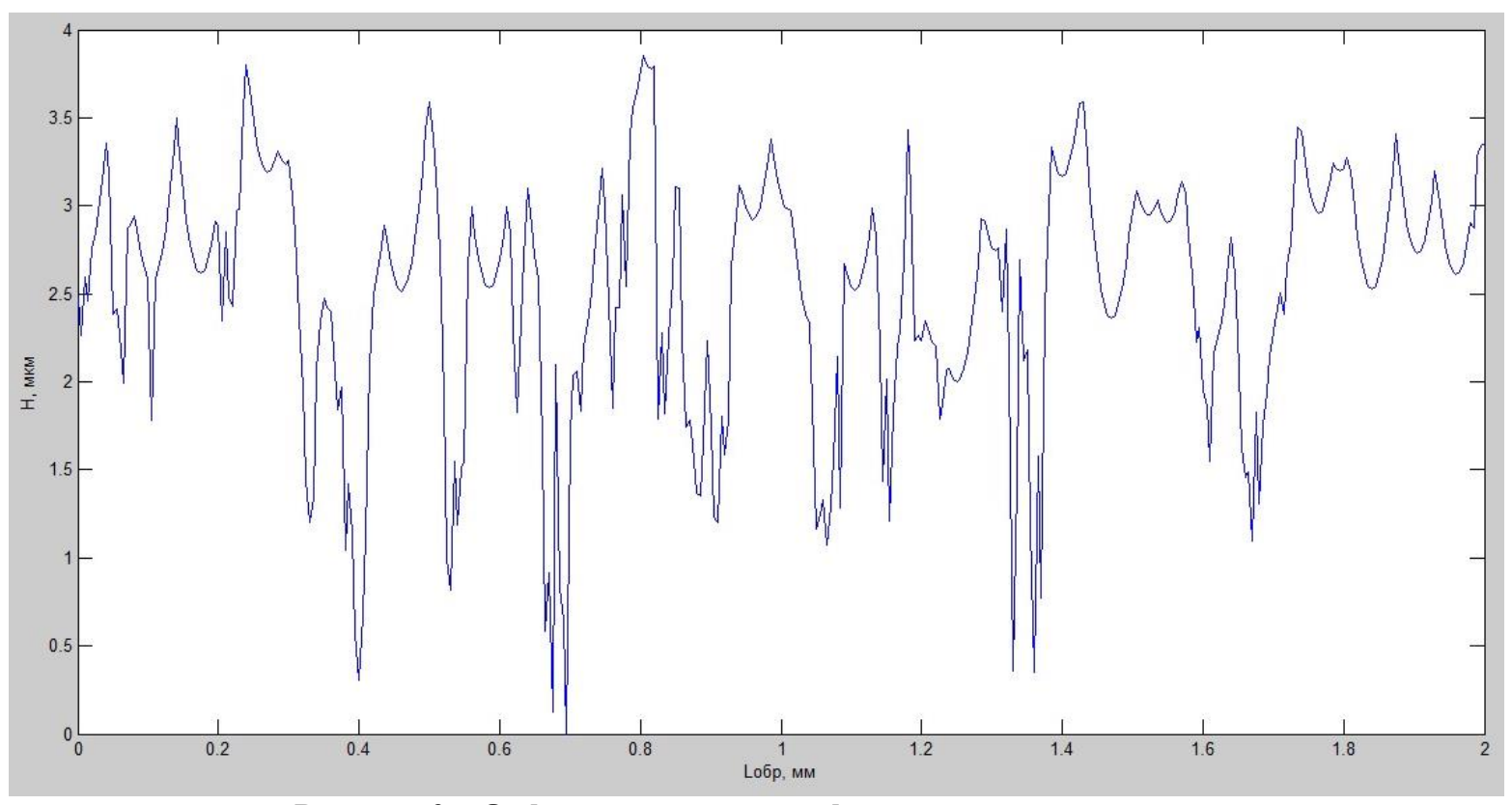

Рисунок 2 - Отфильтрованная профилограмма поверхности

ISPC Generalization of scientific results, 


\begin{tabular}{l|lrl|l|ll} 
& ISRA (India) & $=\mathbf{1 . 3 4 4}$ & SIS (USA) & $=\mathbf{0 . 9 1 2}$ & ICV (Poland) & $=\mathbf{6 . 6 3 0}$ \\
Impact Factor: & ISI (Dubai, UAE) $=\mathbf{0 . 8 2 9}$ & PUHU (Russia) $=\mathbf{0 . 2 3 4}$ & PIF (India) & $=\mathbf{1 . 9 4 0}$ \\
& GIF (Australia) & $\mathbf{0 . 5 6 4}$ & ESJI (KZ) & $=\mathbf{1 . 0 4 2}$ & IBI (India) & $\mathbf{4 . 2 6 0}$
\end{tabular}

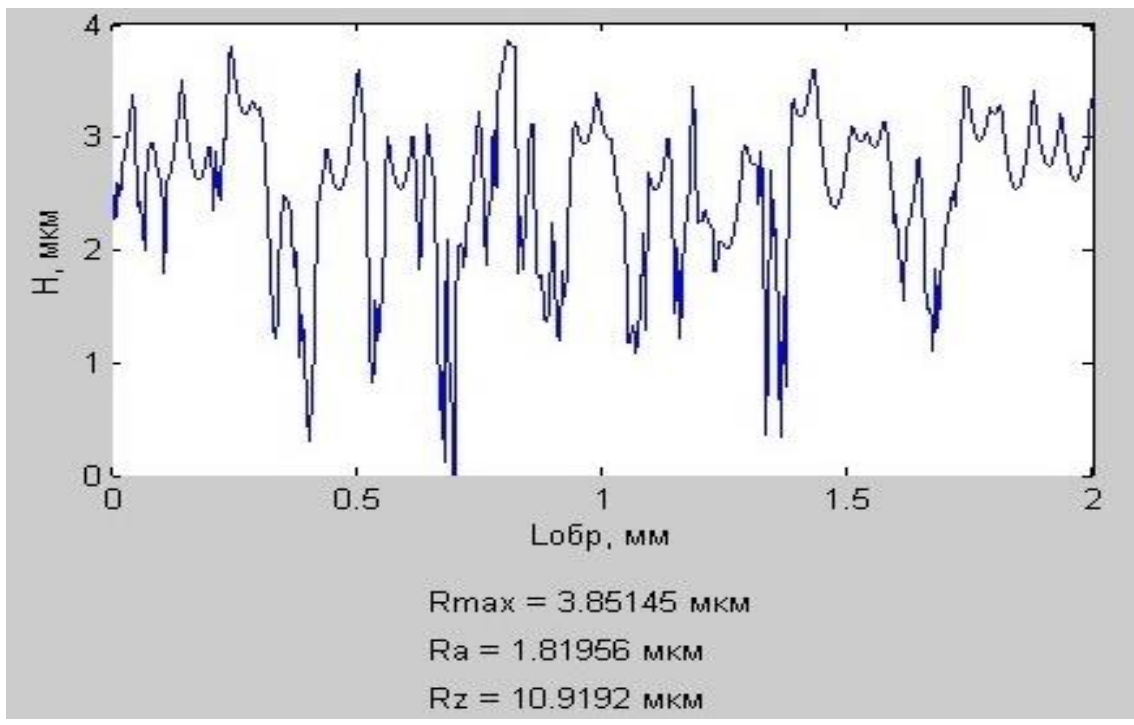

Рисунок 3 - Пример оценки параметров микрогеометрии этапы:

Алгоритм включает следующие основные

1. Ввод данных о параметрах микрогеометрии;

2. Определения дискретности вычислений по осям;

3. Предварительный расчет;

4. Задание исследуемого сечения;

5. Определение двухмерных параметров по трехмерной модели; *.mat.

6. Сохранение массива в файл формата

Wmax - максимальное значение из массива профилограммы поверхности, Msize - длина профилограммы по оси абсцисс, К - массив данных профилограммы.

\section{Conclusion}

Программа позволяет рассчитать двумерные параметры микрогеометрии: $\mathrm{Rmax}, \mathrm{Ra}, \mathrm{Rz}[7,8$, 9].

1. Определение положения средней линии профилограммы:

$$
m=\int_{0}^{l} y^{2} d x=\min
$$

2. Вычисление Ra [1]:

$$
R a=\frac{1}{n} \sum_{i=1}^{n} y_{i}
$$

где $\mathrm{y}_{\mathrm{i}}$ - отклонение точки от средней линии профиля.

3. Вычисление $\mathrm{Rz}$ по 10-ти точкам. Используется сортировка «пузырьком» [10] с нахождением двух массивов с 5 точками максимумов и минимумов профилограммы:

$$
R z=\left(\sum_{i=1}^{5}\left|H_{i \max }\right|+\sum_{i=1}^{5}\left|H_{i \min }\right|\right) / 5
$$

4. Вычисление Rmax:

$$
R \max =\max (K),
$$

где $\mathrm{K}$ - массив точек исследуемого профиля поверхности. 


\begin{tabular}{l|lr|ll|ll} 
& ISRA (India) & $\mathbf{1 . 3 4 4}$ & SIS (USA) & $=\mathbf{0 . 9 1 2}$ & ICV (Poland) & $=\mathbf{6 . 6 3 0}$ \\
Impact Factor: & ISI (Dubai, UAE) $=\mathbf{0 . 8 2 9}$ & PUHЦ (Russia) & $=\mathbf{0 . 2 3 4}$ & PIF (India) & $=\mathbf{1 . 9 4 0}$ \\
& GIF (Australia) & $\mathbf{0 . 5 6 4}$ & ESJI (KZ) & $=\mathbf{1 . 0 4 2}$ & IBI (India) & $=\mathbf{4 . 2 6 0}$ \\
& JIF & $\mathbf{1 . 5 0 0}$ & SJIF (Morocco) & $\mathbf{2 . 0 3 1}$ & & \\
\hline
\end{tabular}

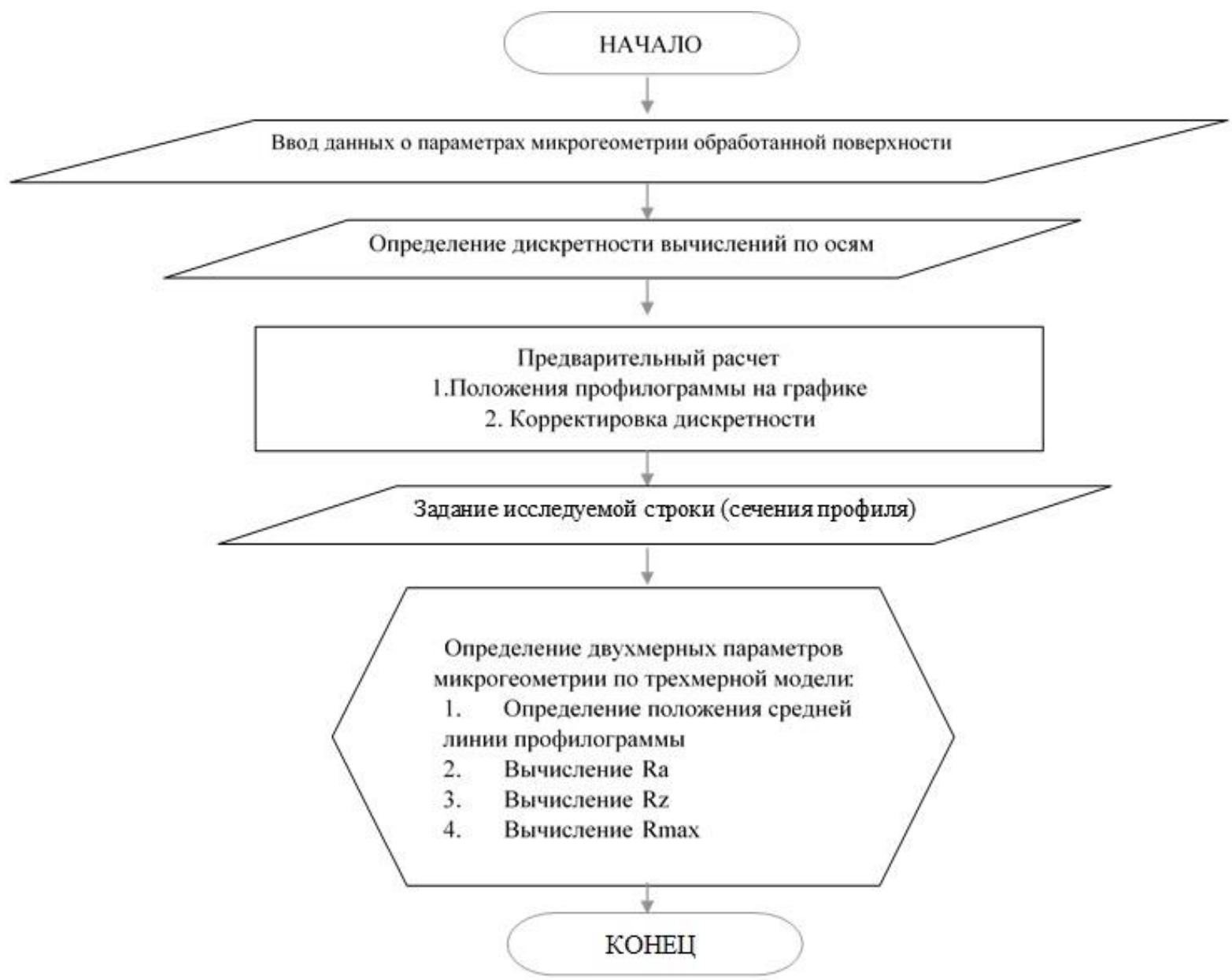

Рисунок 4 - Блок-схема алгоритма.

Данные о поверхности могут быть использованы как отдельно, так и дополнительно оценены численно, например, если планируется характеризовать поверхность с помощью 3D параметров.

\section{References:}

1. Bobrovskij NM, Vil'chik VA, Bokk VV, Bobrovskij IN (2008) Raspredelenie temperatur pri vyglazhivanii shirokim samoustanavlivajushhimsja instrumentom // Izvestija Samarskogo nauchnogo centra Rossijskoj Akademii Nauk. 2008. No. S6. p. 22-29. Available: http://elibrary.ru/item.asp?id=21030520 (Accessed: 23.08.2016).

2. Bobrovskij NM, Barabanov SI, Maksimenko NN, Bobrovskij IN (2008) Tehnicheskie reshenija, primenjaemye pri obrabotke PPD shirokimi samoustanavlivajushhimisja instrumentami // Izvestija Samarskogo nauchnogo centra Rossijskoj Akademii Nauk. 2008. No. S6. - p. 30-36. Available: http://elibrary.ru/item.asp?id=21030499

(Accessed: 23.08.2016).

3. Gorshkov BM, Samohina NS, Remneva OJ (2012) Issledovanie tehnologicheskih sistem precizionnyh gorizontal'nyh koordinatnorastochnyh stankov metodom konechnyh 


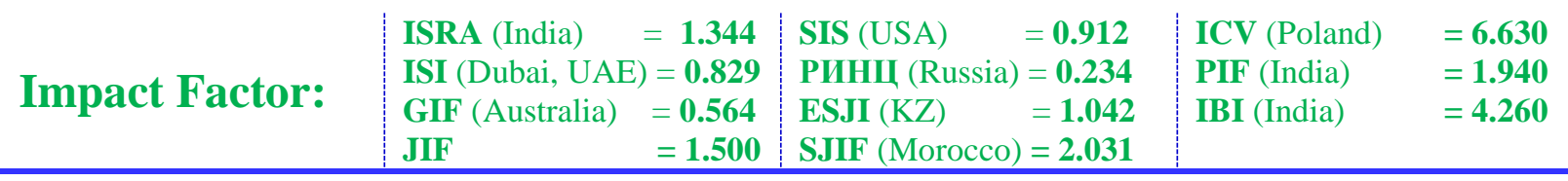

jelementov // Metalloobrabotka. 2012. No 5-6. p. 69-73.

4. Mel'nikov PA, Pahomenko AN, Luk'janov AA (2015) Matematicheskaja model' formirovanija mikrorel'efa shejki vala pri obrabotke almaznym vyglazhivaniem // Vektor nauki Tol'jattinskogo Gosudarstvennogo Universiteta. 2015. No. 2 (32-2). p. 104-111. Available: http://elibrary.ru/download/23129708.pdf (Accessed: 13.11.2016).

5. Brzhozovskij BM, Zaharov OV (2005) Profilirovanie vedushhego kruga pri bescentrovom kruglom shlifovanii cilindricheskih detalej // STIN. 2005. No. 4. p. 12.

6. Zaharov OV, Brzhozovskij BM (2006) Izmerenie otklonenija ot kruglosti $\mathrm{s}$ ispol'zovaniem garmonicheskogo analiza // Kontrol'. Diagnostika. 2006. No. 1. p. 49.
7. (2000) GOST 2.309-73. Oboznachenija sherohovatosti poverhnostej. M.: IPK Izdatel'stvo standartov, 2000. 8 p.

8. (2000) ISO 13565-2:1996. Geometricheskie harakteristiki izdelij (GPS). Struktura poverhnosti: profil'nyj metod. Poverhnosti s poslojnym raspredeleniem funkcional'nyh svojstv. Chast' 2. Harakteristika slojov metodom vydelenija linejnogo uchastka na krivoj procentnogo soderzhanija materiala. BSI, 2000. $30 \mathrm{p}$.

9. (1996) ASME B46.1. Surface Texture (Surface Roughness, Waviness, and Lay). The American Society of Mechanical Engineers, 1996. 120 p.

10. (2016) Bubble sort. URL: https://en.wikipedia.org/wiki/Bubble_sort (Accessed: 10.08.2016). 